\title{
ASCA DEEP SKY SURVEY
}

The Log $N$-Log $S$ relation of faint extragalactic objects and their contribution to the Cosmic X-Ray Background

\author{
Y. OGASAKA \\ NASA Goddard Space Flight Center \\ T. KII, Y. UEDA, T. TAKAHASHI, H. INOUE \\ The Institute of Space and Astronautical Science \\ Y. ISHISAKI \\ Department of Physics, Tokyo Metropolitan University \\ K. OHTA \\ Department of Astronomy, Kyoto University \\ T. YAMADA \\ Astronomical Institute, Tohoku University \\ K. MAKISHIMA \\ Department of Physics, University of Tokyo \\ AND \\ T. MIYAJI, G. HASINGER \\ Astrophysikalisches Institut Potsdam
}

\section{Introduction}

ASCA DSS was intended to carry out unbiased surveys in wide energy range of $0.5-10 \mathrm{keV}$. The strategy of this project is to survey small sky region with extremely high sensitivity reaching to the source confusion limit of $A S C A$ XRT, in contrast to the Large Sky Survey project (Ueda 1996) which covers much larger sky area with relatively shallow exposure.

The regions surveyed and so far studied include Lynx Field ( $75 \mathrm{ksec}$ ), Lockman Hole ( $55 \mathrm{ksec}$ ) and Selected Area 57 (SA57, $250 \mathrm{ksec}$ ). These fields are all known to be less extincted by galactic absorption and have deep previous survey observations in optical and other wavebands.

\section{2. $\log \mathrm{N}-\log \mathrm{S}$ relation}

From the number density distribution of faint sources detected from DSS, we derived 2-10 keV $\log N-\log S$ relation at the limiting flux of $3.80 \times 10^{-14}$ 
$\operatorname{erg~} \mathrm{sec}^{-1} \mathrm{~cm}^{-2}$. This is consistent with the extrapolation of $\log N-\log S$ relations from previous experiments(e.g., Hayashida 1991; Piccinotti et al. 1982). At this flux limit about $40 \%$ of the CXB intensity in the $2-10 \mathrm{keV}$ band is resolved into discrete sources.

We also derived $0.5-2 \mathrm{keV} \log N-\log S$ relation at the flux limit of $9.23 \times 10^{-15} \mathrm{erg} \mathrm{sec}^{-1} \mathrm{~cm}^{-2}$. The number density obtained seems to be slightly larger than ROSAT values(e.g., Hainger et al. (1993)), although it is consistent within the statistical errors and systematic errors due to uncertainties of assumption of spectral index.

\section{Source spectrum}

Averaged spectrum of detected sources was obtained by hardness-ratio study in $2-10 \mathrm{keV}$ band, as $\alpha=0.3 \pm 0.4$ which is consistent with the index of the CXB $(\alpha=0.4)$.

At brighter flux regions, Ginga and HEAO-1 experiments obtained averaged indices of $0.8 \pm 0.1$ and $0.9 \pm 0.1$, respectively. In this flux region, main contributor to the CXB is considered to be Seyfert 1 Galaxies whose mean index is about 0.7. The hardening of the spectrum we observed can be interpreted as a contribution from hard X-ray dominant sources like absorbed AGNs.

The comparison of the source spectrum for quasar and non-quasar candidates was obtained from cross-identification and spectral analysis of sources detected in SA57 with KKC optical quasar catalog (Koo, Kron and Cudworth 1986; Koo and Kron 1988). The averaged $0.5-10 \mathrm{keV}$ band spectral index for samples with KKC identifications is $\alpha=0.63 \pm 0.31$. This is consistent with averaged spectrum of high-z quasars observed with $A S C A$, which is $\alpha=0.61 \pm 0.04(0.5-10 \mathrm{keV}$, Cappi et al. 1997). On the other hand, averaged spectrum of sources with no KKC identifications is hard. If we express this spectrum by absorbed power law, absorption column is estimated as $\mathrm{NH}=(9 \pm 4) \times 10^{21} \mathrm{~cm}^{-2}$ with fixed power law index of $\alpha=0.7$.

Acknowledgements. YO acknowledges the support of the Postdoctoral Fellowships for Research Abroad of the Japan Society for the Promotion of the Science.

\section{References}

Cappi, M. et al. 1997, in the proceedings of " $X$-ray Imaging and Spectroscopy of Cosmic Hot Plasmas", Tokyo, March 1996

Koo, D. C., Kron, R. G., and Cudworth, K. M. 1986, Publ. Astron. Soc. Pacific, 98, 285.

Hasinger, G. et al., 1993, Astron. Astrophys., 275, 1.

Hayashida, K. 1989, Ph. D dissertation of Univ. of Tokyo, ISAS RN 466.

Ogasaka, Y. 1996, Ph. D dissertation of Gakushuin University

Piccinotti, G. et al 1982, Astrophys. J., 253, 485.

Ueda, Y. 1996, Ph. D dissertation of University of Tokyo 